Document downloaded from:

http://hdl.handle.net/10251/62854

This paper must be cited as:

García Meca, C. (2013). Synthesis of low-loss metamaterials with negative index in the visible domain. Modern Physics Letters B. 27(15). doi:10.1142/S0217984913300111.

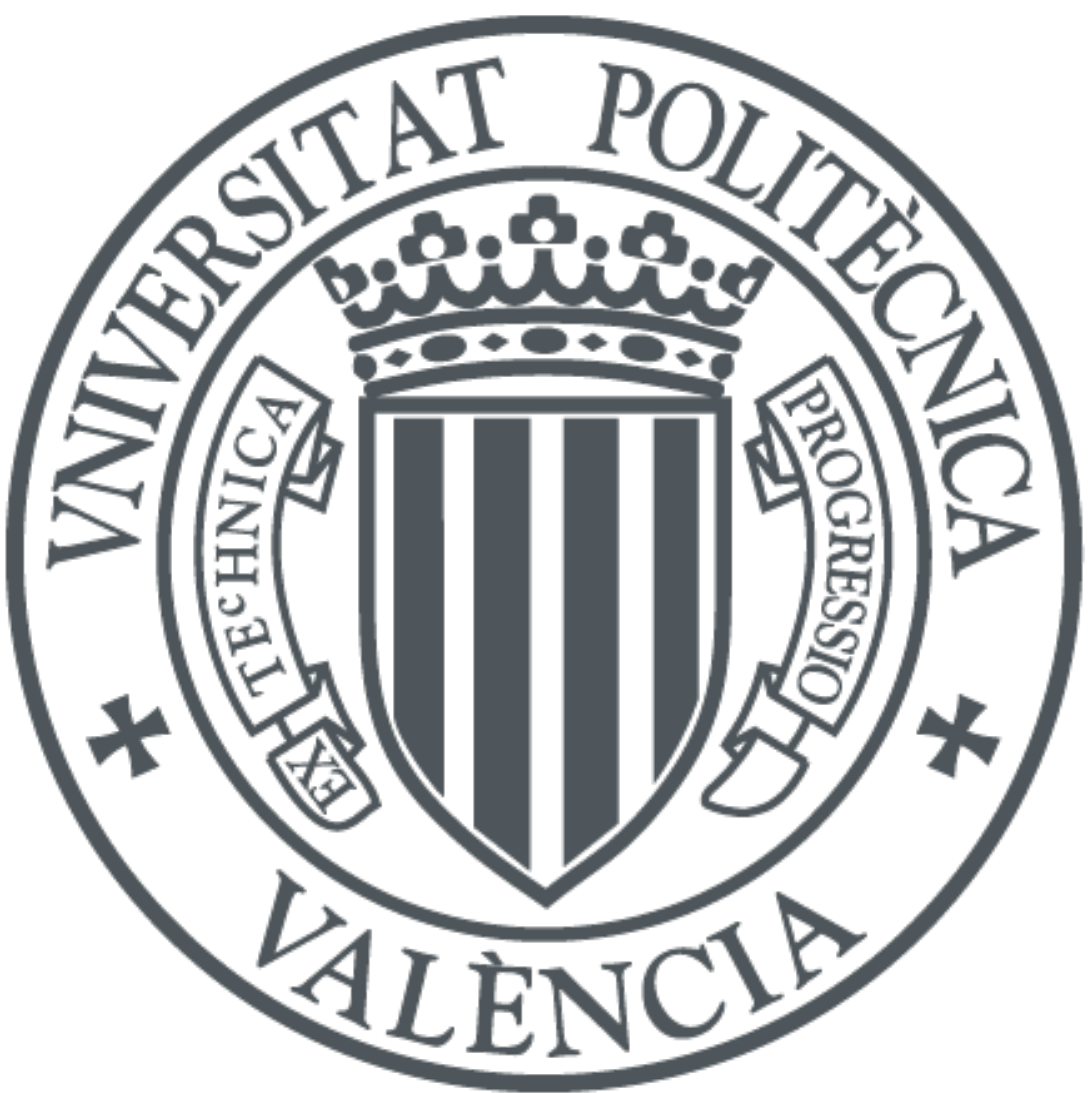

The final publication is available at

http://dx.doi.org/10.1142/S0217984913300111

Copyright World Scientific Publishing

Additional Information 


\title{
SYNTHESIS OF LOW-LOSS METAMATERIALS WITH NEGATIVE INDEX IN THE VISIBLE DOMAIN
}

\author{
CARLOS GARCÍA-MECA \\ Nanophotonics Technology Center, Universitat Politècnica de València, Camino de Vera s/n \\ Valencia, 46022, Spain \\ cargarm2@ntc.upv.es
}

\begin{abstract}
Over the last decade, negative index media have attracted much attention due to their potential applications, specially the possibility of constructing superlenses. However, achieving high-performance negative-index metamaterials at visible frequencies, where this kind of media could find many applications, still remains a challenge. In this article, we provide a brief overview of the main routes for the implementation of metamaterials with negative index in this band, with special focus on the so-called fishnet metamaterial. We pay particular attention to a special fishnet configuration that recently allowed for the experimental demonstration of a low-loss and polarization-insensitive negative-index band in the visible regime.
\end{abstract}

\section{Introduction}

Metamaterials are a relatively new class of man-made electromagnetic materials (the concept was later extended to other fields of physics) that appeared just over a decade ago. [1-4] As opposed to photonic band-gap media,[5] which are based on the phenomenon of diffraction and whose crystalline structure has a periodicity comparable to the wavelength of light, metamaterials can be regarded as effective media characterized by specific electric permittivity and permeability constants (the constitutive parameters), since their lattice period is much smaller than the wavelength. A huge advantage of these artificially structured composites is that, in addition to their chemical composition, they owe their properties to the shape and dimensions of their constituent elements. As a consequence, they can be engineered to possess properties beyond those found in nature. This is the reason why they have been termed metamaterials. These metamedia have brought about a myriad of new devices with amazing properties and are shaping up as a new paradigm in the design of electromagnetic structures.[1-4, 6-11]

The development and impact of metamaterials have been enormous in the last few years. To a large extent, this is due to the discovery in 2000 of the resolving power of negative-refractive-index lenses.[12] Many other applications of negative-index media (NIMs) have been proposed throughout the short history of metamaterials.[6] Among 
them we find the construction of sub-wavelength optical waveguides, [13] the design of low-weight antennas and reflectors,[14] and the improvement of the magnetic resonance imaging technique.[15] Notwithstanding, the possibility of building a so-called superlens $[12]$ stands out from the rest. Let us briefly explain the main advantage of this kind of device.

It is well-known that the resolution of conventional lenses is limited by diffraction. This fact can be easily understood by using the angular-spectrum decomposition of the fields,[16] with which we can express any field distribution $\mathbf{A}(x, y, z)$ as ${ }^{1}$

$$
\begin{aligned}
\mathbf{A}(x, y, z) & =\int_{-\infty}^{\infty} \int_{-\infty}^{\infty} \hat{\mathbf{A}}\left(k_{x}, k_{y}, z\right) e^{i k_{x} x} e^{i k_{y} y} d k_{x} d k_{y}, \\
\hat{\mathbf{A}}\left(k_{x}, k_{y}, z\right) & =\int_{-\infty}^{\infty} \int_{-\infty}^{\infty} \mathbf{A}(x, y, z) e^{-i k_{x} x} e^{-i k_{y} y} d x d y
\end{aligned}
$$

Eq. (2) is the two-dimensional Fourier transform with respect to the spatial variables $x$ and $y$. Thus, $k_{x}=2 \pi f_{x}$ and $k_{y}=2 \pi f_{y}$ are the angular spatial frequencies in each direction, with $f_{x}$ and $f_{y}$ being the spatial frequencies. On the other hand, if we focus on the fields in the plane $z=0$, Eq. (1) expresses the field as an infinite sum of plane waves of the form

$$
\hat{\mathbf{A}}\left(k_{x}, k_{y}, 0\right) e^{i\left(k_{x} x+k_{y} y\right)}
$$

which is known as the angular spectrum of $\mathbf{A}$. In addition, for a time-harmonic variation of the fields, A must satisfy the Helmholtz wave equation

$$
\nabla^{2} \mathbf{A}+k^{2} \mathbf{A}=0
$$

Upon substitution of Eq. (1) into Eq. (4) we find that the following equation must be fulfilled

$$
\left(\frac{\partial^{2}}{\partial z^{2}}+k^{2}-k_{x}^{2}-k_{y}^{2}\right) \hat{\mathbf{A}}\left(k_{x}, k_{y}, z\right)=0
$$

with $k^{2}=k_{x}^{2}+k_{y}^{2}+k_{z}^{2}$, and whose solution is given by

$$
\hat{\mathbf{A}}\left(k_{x}, k_{y}, z\right)=\hat{\mathbf{A}}\left(k_{x}, k_{y}, 0\right) e^{i k_{z} z} .
$$

Therefore, the effect of propagation over a distance $z$ can be obtained by propagating each component of the angular spectrum that distance. Remarkably, the components of $\mathbf{A}(x, y, 0)$ with a spatial periodicity smaller than the wavelength $\lambda$ have an associated plane wave with an imaginary propagation constant $k_{z}=2 \pi\left(1 / \lambda^{2}-f_{x}^{2}-f_{y}^{2}\right)^{1 / 2}$, which is actually evanescent in the $z$ direction. This is the reason why the finer details of

\footnotetext{
${ }^{1} \mathbf{A}$ may represent either the electric field $\mathbf{E}$ or the magnetic field $\mathbf{H}$.
} 
an object (smaller than the wavelength) cannot be resolved by a far-field lens. Plane waves carrying the bulk features of the object propagate through space reaching the lens, while evanescent waves carrying the finer features exponentially decay as we move away from the source. Classical lenses are able to correct the phase delay that each angularspectrum component accumulates with propagation, but cannot restore the amplitude of evanescent waves, which either do not reach the lens or are dramatically attenuated when they reach the focal point.

It has been known for many years that a medium with simultaneously negative permittivity and permeability displays a negative refractive index and that a slab of such a medium also acts as a lens for rays.[17] The big difference between conventional lenses and the NIM slab is that, in addition to restoring the phase of propagating waves, a medium with permittivity and permeability equal to -1 restores the amplitude of evanescent waves, which reach the focus with the same amplitude they had in the object plane. As a result, we obtain a perfect image reconstruction. This is the reason behind the term perfect lens or superlens. This apparent amplification of evanescent waves by a passive device does not violate energy conservation, as these waves do not carry energy. The amplification phenomenon is linked to the excitation of surface waves at the interface between the lens and the surrounding medium, known as surface plasmon polaritons (SPPs).[18, 19]

Undoubtedly, it would be very important to have at our disposal NIMs operating in the visible range, where we can take full advantage of their properties. For instance, the superior imaging ability of NIMs would be essential for visible microscopy, with applications in microelectronics, bio- and nano-technology. It is worth mentioning that the evolution of superlenses gave rise to another interesting kind of lens: the hyperlens. In brief, a hyperlens transforms evanescent components into propagating ones by magnifying the near field pattern, which can then be treated with conventional optics. [9, 20,21] This way the resolution of our far-field system is improved by the magnification factor. Furthermore, transformation optics has led to alternative hyperlens designs.[22, 23]

In addtion to the construction of superlenses, there are other properties of NIMs with potential interesting properties in the visible spectrum. As an example, it has been shown that the inverse Goos-Hänchen effect appearing at the interface between a NIM and a positive-index material could be used to stop light. This effect could be achieved in an optical waveguide in which the core medium has a negative refractive index.[24] Other applications are the possibility of turning the Casimir force from attraction to repulsion and achieving quantum levitation[25] or the use of NIMs to enhance optical gradient forces between dielectric slab waveguides.[26]

Unfortunately, achieving a negative refractive index at visible frequencies has proved to be an extraordinary challenge. $[7,8,10]$ The main reason comes from the difficulty in obtaining strong magnetic responses in this spectral range due to fundamental physical limitations. Additional technological limitations further hinder the construction of magnetic metamaterials if three-dimensional structures with several meta-atoms in the propagation direction are desired.[10, 27]

In this review article we provide a brief overview of some of the most important 
approaches proposed so far for the implementation of metamaterials with negative permeability and negative index in the visible spectrum, with special focus on the so-called fishnet metamaterial. This kind of structure has been widely studied as a means to obtain optical NIMs. We pay particular attention to a special fishnet configuration that has recently allowed us to demonstrate experimentally a low-loss and polarization-insensitive negative-index band in the visible regime.

The article is organized as follows. In sections 2 and 3 we summarize, respectively, the main routes for achieving strong optical magnetic activity and NIMs in the visible spectrum. The fishnet metamaterial is described in depth in section 4. Its physical interpretation as well as suitable fabrication techniques for its practical realization are reviewed. Finally, conclusions are drawn in section 5 .

\section{Artificial magnetism in the visible spectrum}

In a dissipative medium, the double negativity requirement of NIMs (simultaneous negative permittivity and permeability) is replaced by the less stringent condition[28]

$$
\epsilon^{\prime} \mu^{\prime \prime}+\epsilon^{\prime \prime} \mu^{\prime}<0
$$

where $\epsilon=\epsilon^{\prime}+i \epsilon^{\prime \prime}$ and $\mu=\mu^{\prime}+i \mu^{\prime \prime}$ are the medium complex permittivity and permeability. Consequently, a medium with $\epsilon^{\prime}<0$ and high magnetization loss could exhibit a negative refractive index, even if the permeability has a positive real part. However, it is clear from condition (7) that only double-negative NIMs will exhibit low losses. Note that in materials exhibiting magneto-electric couplings, simultaneous negative permittivity and permeability are not necessary to achieve a negative index of refraction, even in the low-loss case. [29, 30]

The first effective medium theories of composites of macroscopic materials started to be developed more than a century ago. One of the oldest and most popular ones is Maxwell-Garnett's formula, which provides the effective permittivity of homogeneous spheres diluted into an isotropic environment as a function of the permittivity of both media and the spheres filling fraction. [31] Of special interest to us are artificial negativepermittivity materials as a means to obtain NIMs. It is relatively easy to achieve this kind of media, since many natural materials exhibit a negative permittivity in a certain spectral range. In fact, most metals present a negative permittivity at frequencies below the so-called plasma frequency, which usually lies in the UV part of the spectrum. As a consequence, tailor-made negative permittivities can be readily obtained by using metallic meta-atoms. For instance, wire media have been extensively studied in the last years as a means to achieve materials with engineered (negative) values of permittivity (see Fig. 1).[32-34]

Artificial magnetism is considerably more difficult to attain, specially at optical frequencies, where natural media display no magnetic response. This is the main challenge when it comes to synthesize low-loss metamaterials exhibiting a negative index of refraction in the visible domain. 


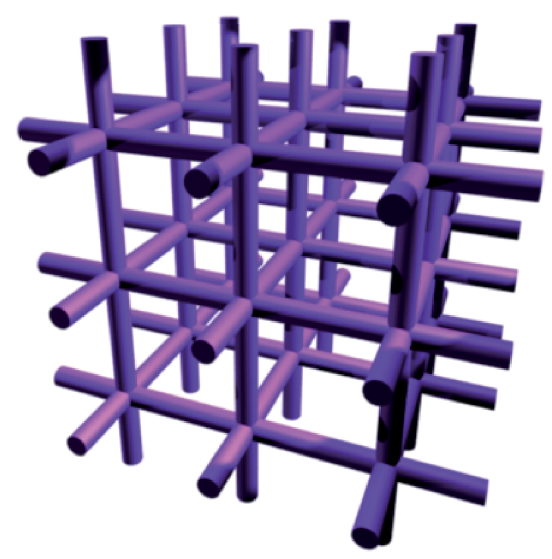

Figure 1: Three-dimensional wire media exhibits an electronic plasma-like behavior with a reduced effective plasma frequency.

The so-called split ring resonator (SRR) was the first meta-atom designed to exhibit an effective negative magnetic permeability (see Fig. 2).[35] Initially, it was designed to display a resonant magnetic response at microwave frequencies. Since then, it has probably become the most widely-used meta-atom for the creation of artificial magnetism. In fact, there exist a number of variants of this structure with different properties.[36, 37].

Apart from the SRR, a variety of structures have been proposed with the aim of obtaining media displaying an effective permeability different from unity. Like in the SRR, magnetic responses are typically achieved by means of metallic structures in which resonant electric currents are induced by the magnetic field of an incident wave.[38-40] Above the resonance frequency, these currents are out of phase with respect to the driving incident wave and generate a magnetic field that opposes the incident one. If the resonance is strong enough, this diamagnetic behavior leads to a negative permeability. It is worth mentioning that a similar behavior appears in composites made up of dielectric materials with high positive values of permittivity.[41-44]

Although initial work was focused on the microwave range, much attention has been put in extending this sought after property to optical wavelengths.[7, 8, 10, 27, 38-40] This effort is justified by the fact that it is in the visible regime where we can make the most of important metamaterial applications in which a magnetic response is required, such as cloaking[45-47], optical security,[48] or imaging, as it is an essential ingredient for low-loss negative-index media.

The first approach towards negative optical magnetism was to take advantage of the scaling properties of Maxwell's equations[5] and scale down the meta-atom size to increase the resonance frequency at which the magnetic resonance occurred. Unfortunately, there exist limits that prevent us from increasing the resonance frequency to an arbitrary high value, since metals stop behaving as perfect conductors in the optical range.[36] Up to date, a number of structures have been proposed to solve this problem. 
(a)

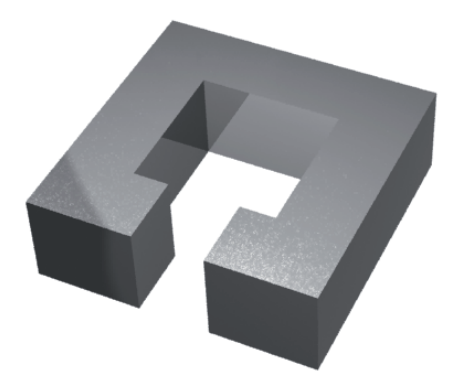

(b)

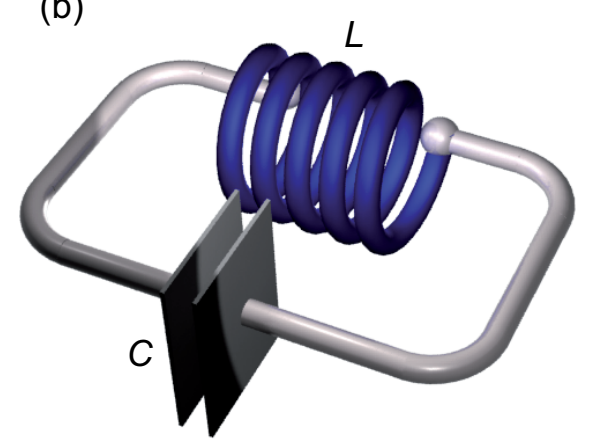

Figure 2: (a) One-cut single split ring resonator (b) The SRR behavior is similar to that of an LC equivalent circuit.

In Fig. 3 we show some of the most important ones.

By modifying the initial geometry of the SRR, the magnetic resonance frequency can be pushed to visible wavelengths. For instance, the introduction of several cuts decreases the SRR equivalent capacitance and consequently the resonance wavelength (see Fig. 3(a)). However, the resonance strength is very week in the visible spectrum and it is extremely difficult to achieve negative permeability values.[36] This problem can be circumvented with arrays of coupled nanostrips[40] or fishnet-like structures[38, 49, 50] as the one shown in Fig. 3(b). We will analyze in depth this metamaterial in next section.

Another possibility is to take advantage of the backward-wave modes supported by metallic coaxial waveguides.[51] A strongly coupled array of this kind of waveguides can be engineered in such a way that a broad angle effective magnetic permeability appears. [52]

All these structures are based on metallic constituents and, although losses can be reduced to reasonable values, for long metamaterial lengths light will experience the unavoidable energy dissipation inherent to metals. A possible way to overcome this drawback is to incorporate a gain medium to the structure.[53] A promising approach relies on exploiting the magnetic resonances that appear in high-index dielectric particles such as semiconductor nanospheres[41-43] or nanocylinders.[44] Up to date, there exist very few experimental works in which a negative permeability band in the visible range has been demonstrated. These experimental demonstrations are mainly based on the fishnet metamaterial (and its complementary structure[40]) or, more recently, on semiconductor nanospheres.[43] 
(a)

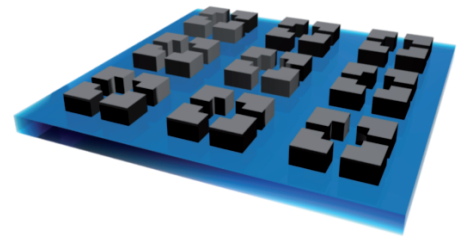

(b)

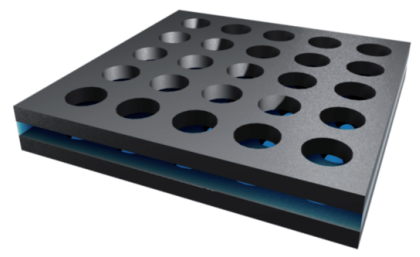

(c)

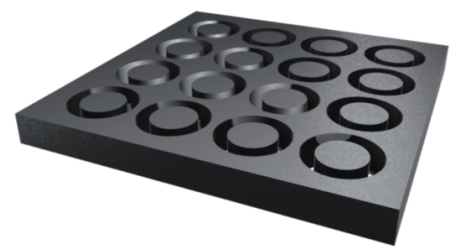

(d)

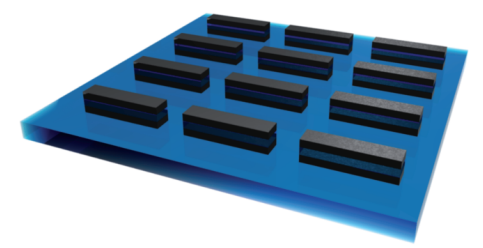

(e)

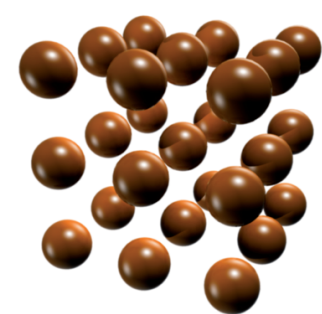

(f)

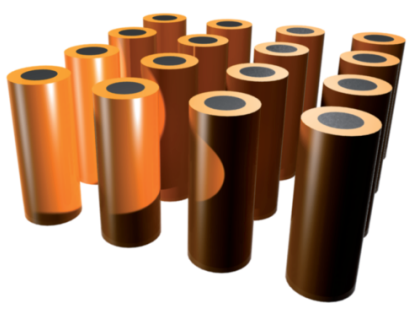

High-index dielectric

Low-index dielectric

Metal

Figure 3: (a) Different metamaterials for achieving magnetic responses at visible frequencies. (a) 4-cut split ring resonators (b) Fishnet structure (c) Array of coaxial metallic waveguides (d) Array of coupled nanostrips (e) High-index spheres (f) High-index cylinders. Here, the cylinders have a metallic core with which a negative index can be achieved. 


\section{Approaches to negative-index media at visible wavelengths}

The structures analyzed in the previous section serve as a starting point to achieve NIMs in the visible spectrum. In order to fulfill the low-loss requirement, an overlapping negative permittivity must be obtained at the same frequency as the negative permeability.

Sometimes, the unit cell must be modified to incorporate the electric feature. In some cases, a careful redesign of the magnetic meta-atom suffices to yield the desired result. For instance, the inclusion of a metallic core in high-dielectric cylinders or spheres provides the sought electric resonance.[44] There exist other proposals based on clusters of plasmonic nanospheres or core-shell nanoparticles with effective negative index or permeability. [54-56]

In other cases, the electric resonance is obtained by adding a second particle, as long as it does not interact strongly with the original ones. SRR media are an example. In fact, except for some special configurations,[57] split ring resonators do not support simultaneous electric and magnetic responses. Negative permittivities in SRR media are usually obtained by adding a second particle such as wires.[58] However, the saturation of the magnetic resonance at optical frequencies renders the SRR unsuitable as a way to achieve NIMs in this range.[36]

Finally, the constituents of some magnetic structures already exhibit electric resonances or a plasma-like behavior that automatically provides the desired negative permittivity. This is the case of properly engineered coaxial waveguide arrays, which in addition to their negative magnetic permeability, also show a negative permittivity at the same frequencies.[52] Something similar occurs with the fishnet structure as we describe in detail below.

\section{Experimental negative-index media based on fishnet-like structures}

The fishnet structure consists of two metallic films separated by a dielectric layer. The whole structure is milled by a periodic array of holes (see Fig. 4). In some configurations the dielectric layer is not milled, although this fact does not alter significantly the behavior of the structure. Depending on the desired properties and employed fabrication techniques, the holes can have different shapes, the most common ones being circular[59] and square.[38] When regarded as an effective medium, this three-layer structure equals a one-atom-thick material in the propagation direction. Each of these atoms is known as a functional layer. An $N$-functional-layer structure can be obtained by stacking individual functional layers separated by a dielectric spacer.[60] In another common configuration, no spacing is left in between neighboring functional layers, in such a way that the metamaterial consists of $2 N+1$ alternating metal and dielectric films.[61] In some special configurations, each functional layer consists of three metallic films separated by two dielectric spacers of different thickness. This kind of supercell can be engineered to obtain an enlarged negative-index bandwidth.[62]

In order to understand better the optical behavior of the fishnet metamaterial, we briefly review its physical interpretation in next section. 


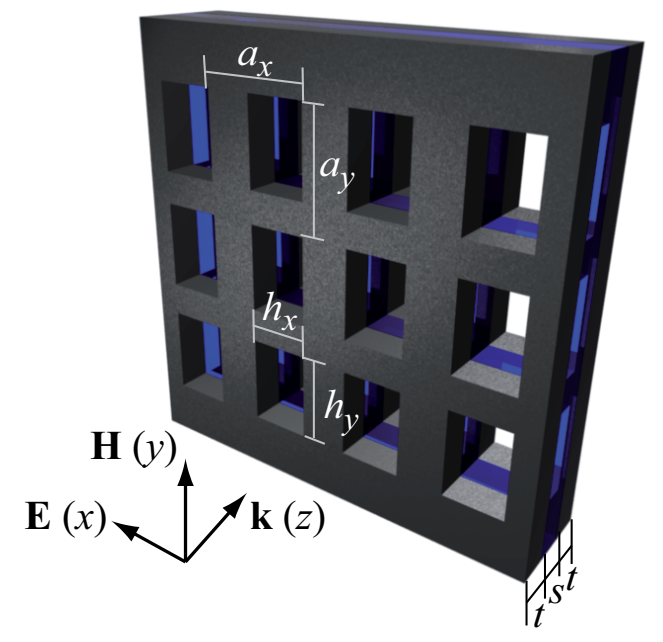

Figure 4: Illustration of the fishnet metamaterial. In the original configurations, the electric field had to be aligned along the thinner strips ( $x$-direction).

\subsection{Physical interpretation}

In the first fishnet configurations, vertical and horizontal strips had a different width. In order to exhibit the desired backward-wave propagation properties, the electric field of incident light was to be oriented along the thinner strips. In that case, the physics of the fishnet structure was explained in terms of the individual response of each kind of strip. The thin strips act as a diluted plasma, thus providing the electric response, while the thick strips are responsible for the magnetic response, as they allowed for the appearance of resonant virtual current loops.[38, 61]

This initial interpretation was further refined by different authors, providing a richer understanding of the fishnet behavior.[63-69] A simple and useful one connects the appearance of backward-wave modes in the fishnet structure with the excitation of SPPs and localized waveguide modes. $[63,65,66]$ This alternative interpretation of the physical mechanisms underlying the fishnet structure opened new perspectives and allowed for the exploitation of certain aspects of this metamaterial that had been previously overlooked. Let us summarize how the electric and magnetic responses of the fishnet metamaterial are explained in this interpretation. For this purpose, we will focus on a standard fishnet configuration studied by several authors. $[66,70]$ In this configuration, the thickness of the metallic layers and dielectric spacer are $t=45 \mathrm{~nm}$ and $s=30 \mathrm{~nm}$, respectively (see Fig. 4). The structure is milled by an array of rectangular holes of sides $h_{x}$ and $h_{y}$, arranged in a square lattice with periodicity $a=a_{x}=a_{y}=600 \mathrm{~nm}$. The transmission for the case $h_{x}=284 \mathrm{~nm}$ and $h_{y}=500 \mathrm{~nm}$ is shown in Fig. 5 . 

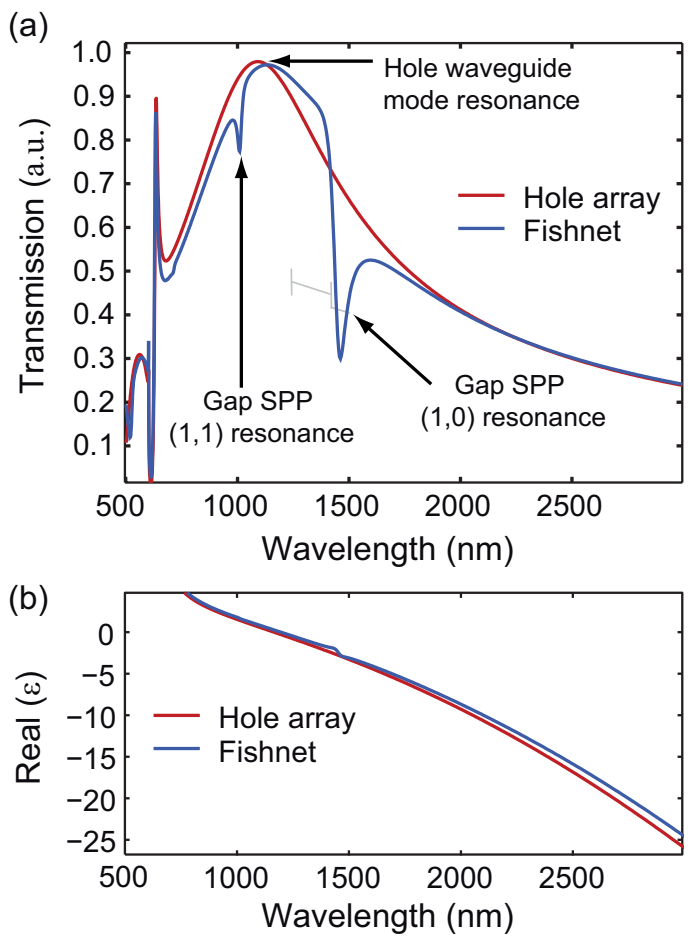

Figure 5: (a) Transmission ( $S_{21}$ parameter) of a fishnet structure with dimensions $t=45$ $\mathrm{nm}, s=30 \mathrm{~nm}, a=a_{x}=a_{y}=600 \mathrm{~nm}, h_{x}=284$, and $h_{y}=500 \mathrm{~nm}$. Transmission of a metallic hole array (same hole size) of thickness $2 t+s$ is also shown. Simulations were performed with CST Microwave Studio, where the metallic films are considered to made up of silver (described by a Drude model with a plasma frequency $\omega_{p}=1.37 \cdot 10^{16}$ and a collision frequency $\omega_{c}=8.5 \cdot 10^{13}$ ), while the permittivity of the dielectric layers is taken to be 1.9. (b) Effective permittivity of both structures.

\subsubsection{Electric response}

It is well-known that waveguide modes can be excited in metallic hole arrays at the mode cut-off wavelength $\lambda_{c}$. [71] Remarkably, this effect is preserved in the fishnet structure, in spite of being made up of two metallic hole arrays separated by a dielectric spacer. $[63,66]$ In the example under consideration, the resonance centered at $\lambda=1.1 \mu \mathrm{m}$ corresponds to one of these localized waveguide modes, which is excited at the cutoff wavelength of the holes for the considered polarization. Note that for a rectangular hole in a perfect metal, $\lambda_{c}$ is $2 h_{x}$. In our case, $h_{x}=500 \mathrm{~nm}$ and therefore, $\lambda_{c}$ should be $1 \mu \mathrm{m}$. However, we are dealing with real metals, in which $\lambda_{c}$ can be significantly increased.[71] In addition, the interplay between adjacent holes may also influence the actual value of $\lambda_{c}$. In Fig. 6 we depict the variation of $\lambda_{c}$ as a function of the incidence angle. Clearly, it has a flat dispersion relation, verifying its localized condition. In addition, the electric field is 
concentrated in the hole (see Fig. 6). The resonance wavelength should not move when changing the thickness of the metal either. This is the case as Fig. 6 also shows. In fact, as $t$ is made higher, Fabry-Perot modes appear at higher frequencies, which are also invariant under angle variations. These Fabry-Perot modes appear when the metal thickness $t$ is an integer multiple of half the wavelength of the mode propagating inside the hole.

(a)
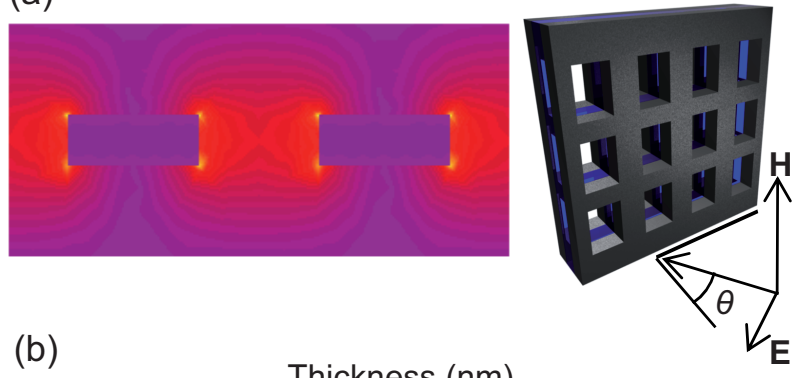

(b)

Thickness $(\mathrm{nm})$

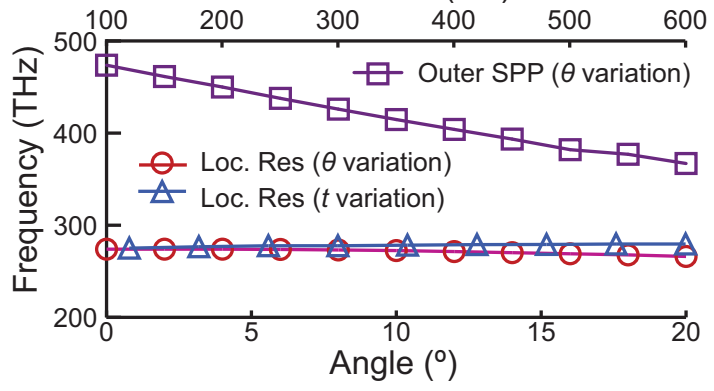

Figure 6: (a) E-field distribution of fishnet structure at the localized resonance frequency. (b) Angle dependence (circles) and $t$ dependence (triangles) of the localized resonance position. Angle dependence of the SPP resonance (squares) is also shown. This resonance exhibits a strong dependence on the incidence angle, as opposed to the localized one.

It can be shown that a metallic single-layer hole array behaves as a bulk metal with a lowered effective plasma frequency determined by the cut-off frequency of the holes.[72] The fishnet metamaterial displays a very similar behavior. In fact, the effective permittivity of the fishnet and of a single-layer metallic hole array of thickness $2 t+s$ are essentially the same, as Fig. 5 shows.[66] Thus, the electric response of the structure can be engineered by varying the shape and size of the holes. Note that the effective parameters (permittivity, permeability and refractive index) of the structure can be obtained from the numerically calculated complex transmission and reflection responses through a standard retrieval algorithm.[73] 


\subsubsection{Magnetic response}

The magnetic response of the fishnet structure can be explained in terms of SPPs. These are hybrid electron-photon excitations that are trapped at the interface between a dielectric (positive-permittivity medium) and a conductor (negative-permittivity medium). While SPPs can propagate along the conductor surface, they are evanescently confined in the perpendicular direction. $[18,19,74]$ This property of SPPs allows us to concentrate electromagnetic fields at the nanoscale by using subwavelength metallic structures. A recent branch of optics known as plasmonics exploits this unparalleled light-concentration ability of metals for a wide range of applications. These include the miniaturization of photonic circuits, modulators and photodetectors, the enhancement of non-linear phenomena, the realization of extremely sensitive biosensors, and the improvement of the efficiency of photovoltaic cells. [19, 75, 76]

A remarkable property of SPPs is that the momentum $k_{\mathrm{FS}}$ of free-space photons is always smaller than the SPP propagation constant $k_{\mathrm{SPP}}$. This prohibits a phase matching between free-space light and SPPs. Hence, SPPs in an ideal semi-infinite medium are nonradiative in nature, i.e., cannot decay by emitting a photon and, conversely, light incident on an ideal surface cannot excite SPPs. Thus, special phase-matching techniques to enhance the momentum of incident light such as grating or prism coupling are required for their excitation.[19, 74] For instance, this mismatch in wave vector can be overcome by periodically patterning the metal surface with grooves or holes with lattice constant $a$. For the simple case of a one-dimensional grating, phase-matching takes place whenever the condition $k_{\mathrm{SPP}}=k_{\mathrm{FS}} \sin \theta \pm n k_{G}$ is fulfilled, where $\theta$ is the angle of incidence, $k_{G}=2 \pi / a$ is the reciprocal vector of the grating, and $n \in \mathbb{N}$. The reverse process also takes place, and so SPPs propagating along a surface modulated with a grating can couple to light and thus radiate.

Since the metallic layers of the fishnet structure are periodically patterned, incident light can excite SPPs on them at certain frequencies. In a fishnet configuration milled by a rectangular hole array with lattice constants $a_{x}$ and $a_{y}$, the phase-matching condition reads

$$
\mathbf{k}_{\mathrm{SPP}}=\mathbf{k}_{\mathrm{FS}}+i \frac{2 \pi}{a_{x}} \mathbf{x}+j \frac{2 \pi}{a_{y}} \mathbf{y},
$$

where $i$ and $j$ are integers. As a first approximation, the propagation constant $k_{\mathrm{SPP}}$ of the plasmonic modes supported by the fishnet structure can be calculated under the assumption that the holes are not present. There exists an analytical expression for the dispersion relation of the different plasmonic modes propagating along the resulting IMIMI structure (I and M stand for insulator and metal, respectively).[65] For instance, the dispersion diagram of the example under study is shown in Fig. 7(b). Two different modes are clearly observed. The one at higher frequencies corresponds to SPPs running along the outer metallic surfaces. The other mode corresponds to a so-called gap SPP: a plasmonic mode existing between both metallic layers.[77]

Let us consider for simplicity the case of normal incidence, for which $\theta$ is zero. From Eq. (8), it is straightforward to obtain the value of $k_{\text {SPP }}$ that corresponds to each plasmonic resonance $(i, j)$. Then, we can obtain the corresponding frequency from the 
dispersion diagram of Fig. 7

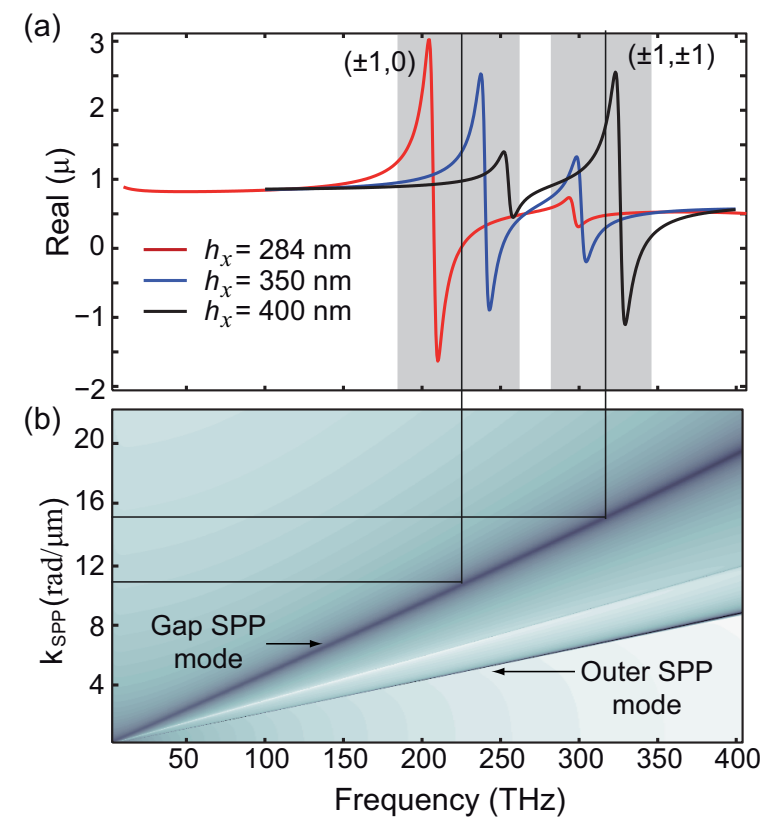

Figure 7: (a) Effective magnetic permeability of the considered fishnet structure for different values of $h_{x}$. The first two magnetic resonances are clearly observed (b) Dispersion relation of the corresponding IMIMI structure.

In our example $a_{x}=a_{y}=a$, so the first SPP resonance appears for $(i, j)=( \pm 1,0)$ or $(i, j)=(0, \pm 1)$, depending on the polarization. This is the magnetic resonance usually employed to achieve a negative permeability in the fishnet structure. At this resonance, there appear virtual current loops that generate magnetic fields that can be out of phase with respect to the incident one. The resonance frequency depends on the structure dimensions $t, s$ and $a$, as well as on the metal and dielectric permittivities. Thus, the frequency at which the negative permeability occurs can be tailored by tuning this parameters.

The effective permeability of our example is depicted in Fig. 7(a). As seen, the frequency of the $(i, j)=( \pm 1,0)$ and $(i, j)=( \pm 1, \pm 1)$ resonances obtained from the dispersion relation [Fig. 7(b)] is in good agreement with the resonances that appear in the effective permeability retrieved from numerical calculations [Fig. 7(a)].

As explained in next section, this interpretation of the fishnet electric and magnetic responses can be used to obtain a double-negative band with some desired properties at visible frequencies. 


\subsection{Low-loss negative-index band at visible wavelengths: design and experimental realization}

According to the previous section, it is possible to obtain a double-negative band at a certain wavelength $\lambda$ as follows. First, we determine the hole dimensions $h_{x}$ and $h_{y}$ of a one-metal-layer hole array such that $\lambda_{c}$ is slightly lower than $\lambda$. This ensures that the permittivity takes moderate negative values at $\lambda$. Next, we choose the proper thickness of the metal and dielectric layers to locate the desired plasmonic resonance at $\lambda .[78]$ As in the first fishnet configurations, it is customary to use the $( \pm 1,0)$ resonance.[79-81] In fact, the fishnet dimensions can be optimized so that a low-loss negative-index band based on this magnetic resonance is achieved in the visible spectrum. [82] However, for polarization independent configurations, this resonance is very weak and the permeability ceases to be negative.[78] This is an important drawback, as polarization independence is a desirable feature in many applications. Fortunately, the $( \pm 1, \pm 1)$ resonance can be used to solve this problem. The reason is that this resonance also has a magnetic nature and that it becomes stronger as we decrease the hole aspect ratio.[65, 78] This can be seen in Fig. 7, where we vary $h_{x}$ keeping $h_{y}$ at a fixed value of $500 \mathrm{~nm}$. As we increase $h_{x}$, the strength of the first magnetic resonance decreases, while the second one displays the opposite behavior.

As a final consideration, note that true three-dimensional metamaterials should be made up of several functional layers, in the same way that natural media consist of multiple atomic layers.[10] As mentioned above, a simple way of achieving a multilayer fishnet metamaterial is by piling alternate metal and dielectric layers so that $2 N+1$ real layers constitute an $N$ functional-layer metamaterial. The effect of stacking several fishnet functional layers is a further loss reduction due to the strong interaction between neighboring layers.[60, 78, 83, 84] Moreover, in strongly coupled systems like this, the effective refractive index changes for low values of $N$.[84] However, the refractive index becomes almost independent of the number of functional layers (as it should happen in a homogeneous medium) for $N>N_{0}$, where $N_{0}$ is usually between 2 and 7.[78, 84]

Therefore, a multiple-layer fishnet structure is highly desired. Following this design methodology, the sought double-negative index band can be located in the visible range. For instance, several three-functional-layer polarization-independent fishnet structures exhibiting a low-loss negative-index band in the visible spectrum were recently designed and verified experimentally. [85] The best results were achieved for a configuration with dimensions $t=35 \mathrm{~nm}, s=15 \mathrm{~nm}, h=h_{x}=h_{y}=250 \mathrm{~nm}$, and $a=a_{x}=a_{y}=400$ $\mathrm{nm}$. To fabricate this metamaterial, a multilayer structure consisting of alternating $\mathrm{Ag}$ and HSQ (hydrogen silsesquioxane) films was deposited. E-beam evaporation and spin coating were employed for the deposition of the Ag and HSQ layers, respectively. Finally, due to its ability to achieve high aspect ratio geometries, focused ion beam milling was used to create the periodic pattern of holes.

The constitutive parameters of such a structure are shown in Fig. 8. A doublenegative band appears at red-light frequencies. It is customary to measure losses through the so-called figure of merit (FOM), defined as FOM $=\left|n^{\prime} / n^{\prime \prime}\right|$, where $n=n^{\prime}+i n^{\prime \prime}$ is the complex effective refractive index. At optical frequencies, FOM values above 3 are 
usually associated with low losses.[70] In this case, the FOM reaches a maximum value of 3.34.[85]

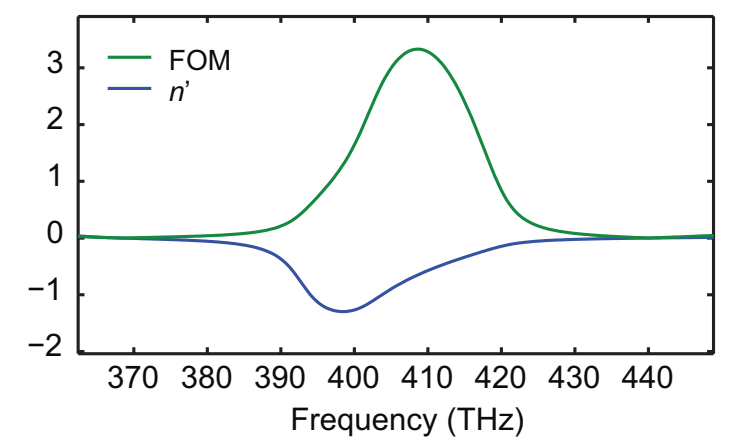

Figure 8: (a) Effective parameters of an experimental three-functional-layer fishnet structure.

Finally, it is worth stressing that, although focused ion beam milling is a useful technique to conduct proof-of-concept experiments, it is not suitable for large-scale manufacturing. In this sense, other approaches such as nanoimprint lithography $[86,87]$ or nanotransfer printing[88] provide a better performance. These techniques are appropriate for fabricating fishnet-like structures such as the one studied here, even over flexible substrates.[88]

\section{Conclusion}

In summary, we have reviewed the main routes for synthesizing metamaterials with optical magnetic activity and low-loss negative index of refraction in the visible spectrum. Up to date, the only experimental demonstrations of double-negative index bands have been achieved with fishnet metamaterials. We have described the main physical mechanisms underlying the electric and magnetic responses of this structure. In addition, we have shown how the knowledge of these mechanisms can be used to obtain a low-loss negative index of refraction at visible wavelengths. Finally, suitable fabrication techniques for implementing the fishnet metamaterial have been briefly reviewed.

We can conclude that, although losses are considerably reduced in optimized configurations of this structure, they are still too large for some applications. In addition, fishnet-like structures are not suitable for achieving isotropic negative-index bands, since they either show an anisotropic behavior[89] or cannot be defined as effective media for off-normal angles of incidence.[64] Therefore, other approaches should be developed for this purpose. For instance, core-shell metal-dielectric structures may provide a good alternative both to further reduce energy dissipation as well as to achieve isotropy, at least in two dimensions.[44]

Nonetheless, the properties of current fishnet designs under normal incidence can 
be used for interesting applications. For instance, negative refraction was observed in a fishnet prism at optical frequencies.[83] An interesting example is given by a recent work that takes advantage of the unusual dispersive features of the negative-index band associated with the fishnet second-order magnetic resonance to control the propagation velocity of a femtosecond laser pulse.[90] In that work, it is shown that subluminal and superluminal pulse propagation velocities can be achieved and tuned just by changing the initial pulse chirp. As a final example, the above-mentioned second-order resonance was used in other work to demonstrate subpicosecond optical switching experimentally.[91]

\section{Acknowledgments}

The author would like to thank R. Ortuño for useful discussions. Financial support from project CONSOLIDER EMET CSD2008-00066 is also acknowledged.

\section{References}

[1] D. R. Smith, J. B. Pendry, and M. C. K. Wiltshire, Science 305 (2004) 788.

[2] H Chen, C. T. Chan, and P. Sheng, Nature Photon. 9 (2010) 387.

[3] M. Wegener and S. Linden, Shaping optical space with metamaterials, Phys. Today 63 (2010) 32.

[4] Y. Liu and X. Zhang, Chem. Soc. Rev. 40 (2011) 2494.

[5] J. D. Joannopoulos, Photonic Crystals (Princeton University Press, 1995)

[6] V. G. Veselago and E. E. Narimanov, Nature Mater. 5 (2006) 759.

[7] C. M. Soukoulis, S. Linden, and M. Wegener, Science 315 (2007) 47.

[8] V. M. Shalaev, Nature Photon. 1 (2007) 41.

[9] X. Zhang and Z. Liu, Nature Mater. 7 (2008) 435.

[10] C. M. Soukoulis and M. Wegener, Nat. Photonics 5 (2011) 523.

[11] C. M. Watts, X. Liu, and W. J. Padilla, Adv. Mater. 24 (2012) OP98.

[12] J. B. Pendry, Phys. Rev. Lett. 85 (2000) 3966.

[13] A. Alù and N. Engheta, IEEE Trans. Microwave Theory Tech. 52 (2004) 199.

[14] C. G. Parazzoli, R. B. Greegor, J. A. Nielsen, M. A. Thompson, K. Li, A. M. Vetter, and M. H. Tanielian, App. Phys. Lett. 84 (2004) 3232.

[15] M. C. K. Wiltshire, J. B. Pendry, I. R. Young, D. J. Larkman, D. J. Gilderdale, and J. V. Hajnal, Science 291 (2001) 849. 
[16] J. W. Goodman, Introduction to Fourier Optics (Roberts \& Company Publishers, 2005)

[17] V. G. Veselago, Sov. Phys. Usp. 10 (1968) 509.

[18] H. Raether, Surface Plasmons on Smooth and Rough Surfaces and on Gratings, (Springer, 1988)

[19] S. Maier, Plasmonics. Fundamentals and applications (Springer, 2007)

[20] Z. Liu, H. Lee, Y. Xiong, C. Sun, and X. Zhang, Science 315 (2007) 1686.

[21] I. Smolyaninov, Y.-J. Hung, and C. C. Davis, Science 315 (2007) 1699.

[22] A. V. Kildishev and E. E. Narimanov, Opt. Lett. 32 (2007) 3432.

[23] C. García-Meca, M. M. Tung, J. V. Galán, R. Ortuño, F. J. Rodríguez-Fortuño, J. Martí, and A. Martínez, Opt. Express 19 (2011) 3562.

[24] K. L. Tsakmakidis, A. D. Boardman, and O. Hess, Nature 450 (2007) 397.

[25] U. Leonhardt and T. G. Philbin, New J. Phys. 9 (2007) 254.

[26] V. Ginis, P. Tassin, C. M. Soukoulis, and I. Veretennicoff, Phys. Rev. Lett. 110 (2013) 057401.

[27] C. M. Soukoulis and M. Wegener, Science 330 (2010) 1633.

[28] R. A. Depine and A. Lakhtakia, Microwave Opt. Tech. Lett. 41 (2004) 315.

[29] J. B. Pendry, Science 306 (2004) 19.

[30] J. Kästel, M. Fleischhauer, S. F. Yelin, and R. L. Walsworth, Phys. Rev. Lett. 99 (2007) 073602.

[31] J. C. Maxwell-Garnett, Philos. Trans. R. Soc. London, Ser. A 203 (1904) 385.

[32] J. B. Pendry, A. J. Holden, W. J. Stewart, and I. Youngs, Phys. Rev. Lett. 76 (1996) 4773.

[33] M. G. Silveirinha, Phys. Rev. E 73 (2006) 046612.

[34] M. G. Silveirinha, Phys. Rev. B 79 (2009) 035118.

[35] J.B. Pendry, A. J. Holden, D. J. Robbins, and W. J. Stewart, IEEE Trans. Microwave Theory Tech. 47 (1999) 2075.

[36] J. Zhou, T. Koschny, M. Kafesaki, E. N. Economou, J. B. Pendry, and C. M. Soukoulis, Phys. Rev. Lett. 95 (2005) 223902.

[37] A. K. Sarychev, G. Shvets, and V. M. Shalaev, Phys. Rev. E 73 (2006) 36609. 
[38] S. Zhang, W. Fan, K. J. Malloy, S. R. J. Brueck, N. C. Panoiu, and R. M. Osgood, Opt. Express 13 (2005) 4922.

[39] Z. Huang and J. Xue and Y. Hou and J. Chu and D. H. Zhang, Phys. Rev. B 74 (2006) 193105.

[40] W. Cai, U. K. Chettiar, H.-K. Yuan, V. C. de Silva, A. V. Kildishev, V. P. Drachev, and V. M. Shalaev, Opt. Express 15 (2007) 3333.

[41] M. S. Wheeler, J. S. Aitchison, and Mohammad Mojahedi, Phys. Rev. B 72 (2005) 193103.

[42] R Paniagua-Domínguez, F López-Tejeira, R Marqués, and J. A. Sánchez-Gil, New J. Phys. 13 (2011) 123017.

[43] A. I. Kuznetsov, A. E. Miroshnichenko, Y. H. Fu, J. Zhang, and B. Lukyanchuk, Sci. Rep. 2 (2012) 492.

[44] R. Paniagua-Domínguez, D. R. Abujetas, and J. A. Sánchez-Gil Sci. Rep. 3 (2013) 1507.

[45] J. B. Pendry, D. Schurig, and D. R. Smith, Science 312 (2006) 1780.

[46] U. Leonhardt and T. G. Philbin, New J. Phys. 8 (2006) 247.

[47] W. Cai, U. K. Chettiar, A. V. Kildishev, and V. M. Shalaev, Nat. Photonics 1 (2007) 224.

[48] A. Martínez, C. García-Meca, R. Ortuño, F. J. Rodríguez-Fortuño, and J. Martí, App. Phys. Lett. 94 (2009) 251106.

[49] S. Zhang, W. Fan, N. C. Panoiu, K. J. Malloy, R. M. Osgood, and S. R. J. Brueck, Phys. Rev. Lett. 95 (2005) 137404.

[50] M. Beruete, M. Sorolla, and I. Campillo, Opt. Express 14 (2006) 5445.

[51] F. J. Rodríguez-Fortuño, C. García-Meca, R. Ortuño, J. Martí, and A. Martínez, Opt. Lett. 34 (2009) 3325.

[52] S. P. Burgos, R. de Waele, A. Polman, and H. A. Atwater, Nat. Mater. 9 (2010) 407.

[53] S. Xiao, V. P. Drachev, A. V. Kildishev, X. Ni, U. K. Chettiar, H.-K. Yuan, and V. M. Shalaev, Nature 466 (2010) 735.

[54] A. Alù and A. Salandrino and N. Engheta Opt. Express 14 (2006) 1557.

[55] C. Rockstuhl and F. Lederer and C. Etrich and T. Pertsch and T. Scharf, Phys. Rev. Lett. 99 (2007) 017401. 
[56] C. R. Simovski and S. A. Tretyakov, Phys. Rev. B 79 (2009) 045111.

[57] F. J. Rodríguez-Fortuño and C. García-Meca and R. Ortuño and A. Martínez and J. Martí, Phys. Rev. B 79 (2009) 75103.

[58] R. A. Shelby, D. R. Smith, and S. Schultz, Science 292 (2001) 77.

[59] M. Navarro-Cía, C. García-Meca, M. Beruete, A. Martínez, and M. Sorolla, Opt. Lett. 36 (2011) 4245.

[60] S. Zhang, W. Fan, N. C. Panoiu, K. J. Malloy, R. M. Osgood, and S. R. Brueck, Opt. Express 14 (2006) 6778.

[61] G. Dolling, M. Wegener S. Linden, Opt. Lett. 32 (2007) 551.

[62] R. Ortuño and C. García-Meca and F. J. Rodríguez-Fortuño and A. Martínez, Opt. Lett. 35 (2010) 4205.

[63] A. Mary, S. G. Rodrigo, F. J. Garcia-Vidal, and L. Martin-Moreno, Phys. Rev. Lett. 101 (2008) 103902.

[64] C. Rockstuhl, C. Menzel, T. Paul, T. Pertsch, and F. Lederer, Phys. Rev. B 78 (2008) 155102.

[65] R. Ortuño, C. García-Meca, F. J. Rodríguez-Fortuño, J. Martí, and A. Martínez, Phys. Rev. B 79 (2009) 75425.

[66] C. García-Meca, R. Ortuño, F. J. Rodríguez-Fortuño, J. Martí, and A. Martínez, Opt. Express 17 (2009) 6026.

[67] R. Marqués, L. Jelinek, F. Mesa, and F. Medina, Opt. Express 17 (2009) 11582.

[68] J. Yang, C. Sauvan, H. T. Liu, and P. Lalanne, Phys. Rev. Lett. 107 (2011) 043903.

[69] V. Torres, P. Rodríguez-Ulibarri, M. Navarro-Cía, and M. Beruete, Appl. Phys. Lett. 101 (2012) 244101.

[70] G. Dolling, C. Enkrich, M. Wegener, C. M. Soukoulis, and S. Linden, Opt. Lett. 31 (2006) 1800.

[71] R. Gordon and A. Brolo, Opt. Express 13 (2005) 1933.

[72] J. B. Pendry, L. Martín-Moreno, and F. J. García-Vidal, Science 305 (2004) 847.

[73] X. Chen, T. M. Grzegorczyk, B.-I. Wu, J. Pacheco, and J. A. Kong, Phys, Rev. E 70 (2004) 016608.

[74] W. L. Barnes, A. Dereux, and T. W. Ebbesen, Nature 424 (2003) 824.

[75] J. A. Schuller, E. S. Barnard, W. Cai, Y. C. Jun, J. S. White, and M. L. Brongersma, Nat. Mater. 9 (2010) 193. 
[76] M. L. Brongersma and V. M. Shalaev Science 328 (2010) 440.

[77] E. N. Economou, Phys. Rev. 182 (1969) 539.

[78] C. García-Meca, R. Ortuño, F. J. Rodríguez-Fortuño, J. Martí, and A. Martínez, Opt. Lett. 34 (2009) 1603.

[79] G. Dolling, M. Wegener, C. M. Soukoulis, and S. Linden, Opt. Lett. 32 (2007) 53.

[80] U. K. Chettiar, A. V. Kildishev, H.-K. Yuan, W. Cai, S. Xiao, V. P. Drachev, and V. M. Shalaev, Opt. Lett. 32 (2007) 1671.

[81] S. Xiao, U. K. Chettiar, A. V. Kildishev, V. P. Drachev, and V. M. Shalaev, Opt. Lett. 34 (2009) 3478.

[82] J. Zhou, T. Koschny, and C. M. Soukoulis Opt. Express 16 (2008) 11147.

[83] J. Valentine, S. Zhang, T. Zentgraf, E. Ulin-Avila, D. A. Genov, G. Bartal, and X. Zhang, Nature 455 (2008) 376.

[84] J. Zhou, T. Koschny, M. Kafesaki, and C. M. Soukoulis, Phys. Rev. B 80 (2009) 035109.

[85] C. García-Meca, J. Hurtado, J. Martí, A. Martínez, W. Dickson, and A. V. Zayats, Phys. Rev. Lett. 106 (2011) 067402.

[86] I. Bergmair, B. Dastmalchi, M. Bergmair, A. Saeed, W. Hilber, G. Hesser, C. Helgert, E. Pshenay-Severin, T. Pertsch, E. B. Kley, U. Hübner, N. H. Shen, R. Penciu, M. Kafesaki, C. M. Soukoulis, K. Hingerl, M. Muehlberger, and R. Schoeftner, Nanotechnology 22 (2011) 325301.

[87] T. W. H. Oates, B. Dastmalchi, G. Isic, S. Tollabimazraehno, C. Helgert, T. Pertsch, E.-B. Kley, M. A. Verschuuren, I. Bergmair, K. Hingerl, and K. Hinrichs, Opt. Express 20 (2012) 11166.

[88] D. Chanda, K. Shigeta, S. Gupta, T. Cain, A. Carlson, A. Mihi, A. J. Baca, G. R. Bogart, P. Braun, and J. A. Rogers Nat. Nanotechnol. 6 (2011) 402.

[89] C. Menzel, R. Alaee, E. Pshenay-Severin, C. Helgert, A. Chipouline, C. Rockstuhl, T. Pertsch, and F. Lederer Opt. Lett. 37 (2012) 596.

[90] V. Yu. Fedorov and T. Nakajima, Phys. Rev. Lett. 107 (2011) 143903.

[91] K. M. Dani, Z. Ku, P. C. Upadhya, R. P. Prasankumar, S. R. J. Brueck, and A. J. Taylor, Nano Lett. 9 (2009) 3565. 